\title{
Editoríal.
}

\section{THE VALUE OF HUMAN EVIDENCE}

H UMAN evidence, the reporting to us by another individual of events which he has witnessed or of experiences through which he has passed or is passing, is a process of fundamental importance in the economy of civilized life, and upon it much of our knowledge is necessarily built. In the law courts it stands out as the paramount factor upon which the whole procedure of justice is based, but it plays a part in every sphere of human activity, and not least in medicine. In every medical case our opinion may be partly formed upon facts which are not or cannot be observed, but which are communicated to us by the patient, and in those departments of medicine where the symptoms are mainly of a subjective character our dependence upon facts of this order reaches an extreme degree. In the psychoneuroses, for example, the phenomena which constitute the clinical picture of the disorder are largely composed of subjective experiences reported to us by the patient, and the accuracy of our case description is necessarily dependent upon the accuracy and reliability of this report. Moreover, the conceptions and theories of the psychopathologist are built up by the analysis and coordination of the reported subjective experiences of the patient, and their validity must inevitably rest upon the same foundation. It is clear, therefore, that both the lawyer and the psychopathologist are vitally interested in the problem of the reliability of evidence, but the psychopathologist has a double interest in that he is not only concerned with the reliability of evidence as a platform upon which he must build his knowledge, but it also falls to him to investigate the factors underlying aberrations from an accurate report and to evaluate the mental processes which produce those aberrations.

To the uninstructed mind the reliability of evidence is a simple matter. A witness is either giving an accurate description of the facts, or he is lying, and the reliability of evidence is measured entirely by the moral worth of the witness. In spite of its almost obvious psychological falsity this naïve view has persisted with remarkable tenacity even in quarters where some knowledge of the real facts might reasonably be expected, and it can be as easily observed in the procedure of the modern law court as in the pseudoscientific writing characteristic of much 
modern psychological literature. Yet there are now at our disposal a great number of facts, both of observation and experiment, which throw a flood of light upon the problem of the reliability of evidence and enable us not only to appreciate the existence of the problem, but to form a notion of the factors and mechanisms upon which reliability depends.

At the beginning of the present century a number of experimental researches were carried out by psychologists, particularly by Stern and his school at Breslau, which decisively disposed of the view that veracity and honesty were interchangeable terms. These experiments consisted essentially in presenting to a witness a series of events, and then requiring him to furnish a report of what he had observed. The results showed that not only were facts omitted in the report, but that many were perverted, and that this applied to an appreciable percentage of facts to whose accuracy the witness was prepared to swear. In one series of experiments the coefficient of accuracy in sworn testimony was under 90 per cent., that is to say, 10 per cent. of the facts sworn to were erroneous. If the report was obtained by questioning the witness instead of merely allowing him to narrate his experiences, the range of facts remembered was increased, but the coefficient of accuracy greatly diminished. If the time interval between the event and the report was increased, both the range and accuracy were diminished, but the assurance of the witness in the truth of his report remained relatively constant. In other words, it was experimentally demonstrated that evidence given with the best knowledge and honesty is by no means necessarily true, that perversion and distortion are appreciable even in the case of facts of whose accuracy the witness is absolutely certain, and that the inaccuracy increases with lapse of time and if methods of questioning are employed, although the belief of the witness in the reliability of his statements is relatively unaffected.

The importance of these findings is obviously very great, but the writings of Stern and his school do not throw much light upon the causal factors and mechanisms whereby the deviations from accuracy in evidence are produced. If we turn to pathology, however, distortion of evidence is a common phenomenon, and the distortion is here so gross that the elucidation of the causal processes is considerably facilitated. It then becomes apparent that evidence, so far from being dependent upon the integrity of a simple faculty of ' memory,' is the end term of a series of processes in every one of which distortion may occur by the action of a multiplicity of causes. In this series of processes we may distinguish perception, registration, conservation, and reproduction, and pathology presents to us numerous examples of perversion in each of them. Perception may be distorted by hallucinations and illusions, registration by the existence of morbid states such as the emotional apathy of dementia præcox, conservation by the organic changes occurring 
in senile and other dementias, and reproduction by the factors which produce hysterical amnesias and confabulations, delusional memories, and the remarkable fabrications of pseudologia phantastica. In all these instances the essential feature is that the evidence is distorted by factors entirely independent both of the actual facts observed and of the volition and moral integrity of the patient. These factors vary in the different conditions mentioned, but they may be said to consist generally either in the endogenous circumstances provided by the patient's emotions, interests, and beliefs, or in the application to him of external forces such as suggestion. There can be no doubt, moreover, that many of these disturbing factors are at work in the normal mind, although obviously their action is more restrained than in the pathological cases, and that the perversions of evidence noted in the Breslau experiments were due to causes of the same kind as those responsible for the gross confabulations met with in definite mental disorder.

These considerations obviously have practical bearings of the utmost importance. It is clear that the legal procedure of examination and cross-examination of witnesses introduces so many perverting factors as to render it a very imperfect method of eliciting the truth in the absence of objective controls, and that the questioning of witnesses with regard to events which happened years previously can only produce grossly inaccurate results, even when the evidence is given with entire honesty and assurance. These imperfections are necessarily present, and probably no alteration in procedure will remove them, but it would at least seem desirable that the Courts should be constantly alive to the existence of the psychological facts, and to the consequences which flow from them.

In psychology, again, as in every other branch of science where we are mainly dependent upon human evidence for our facts and objective controls are rarely available, it is of cardinal importance that the evidence should be scrutinized inexorably, and every possible factor of perversion taken carefully into account. It has to be recognized that the method by which the clinical facts are elicited in the psychoneuroses may itself induce a considerable degree of alteration in those facts, especially in the course of a long-continued investigation, and that the final picture we believe ourselves to have observed may in part be of the nature of an artefact. For it is the foundation of psychopathological theories in facts of observation which requires strengthening, and if this could be stabilized we might reasonably hope for the building of a solid structure upon it. 